of RA in group I was 35.0 years, in group II - 66.2 years.; RA duration was 14.4 years and 4.6 years, respectively. Long-term history of glucocorticoids use (for more than 3 months) was observed in $50 \%$ of patients in group I, and in $42 \%$ of patients in group II.

Results: 40 (18\%) patients in group I and 17 (26\%) patients in group II had fragility fractures. Among patients with fragility fracture, $23(57,5 \%)$ patients in group I and 6 (35\%) patients in group II received glucocorticoid therapy for more than 3 months. Two or more fractures in history had $16(40 \%)$ in group I, and 3 $(18 \%)$ in group II

Conclusion: The frequency of fragility fracture in the study groups was comparable ( $p>0.05)$, despite the age of patients. But, the frequency of refractures was higher in patients with RA-onset at young age, which, apparently, is a consequence of long RA disease duration and use of glucocorticoids.

Disclosure of Interests: None declared

DOI: 10.1136/annrheumdis-2020-eular.1789

\section{SAT0081 \\ FOOTPRINT OF THE BRAIN-DERIVED NEUROTROPHIC FACTOR ON PAIN AND MOOD PERCEPTION OF PATIENTS WITH RHEUMATOID ARTHRITIS}

L. Leifsdottir ${ }^{1}$, C. Wasen ${ }^{1}$, M. C. Erlandsson ${ }^{1}$, K. M. Andersson ${ }^{1}$, R. Heckemann'2, M. I. Bokarewa ${ }^{1} .{ }^{1}$ Sahlgrenska Academy at University of Gothenburg, Department of Rheumatology and Inflammation Research, Göteborg, Sweden; ${ }^{2}$ University of Gothenburg, Department of Radiation Physics, Göteborg, Sweden

Background: Depression and cognitive impairment have been frequently reported in rheumatoid arthritis (RA) (1). Studies of the molecular mechanisms behind these phenomena attract increasing attention. We previously reported that signaling through the insulin-like receptor is impaired in RA and has consequences for pain processing (2).

Objectives: We investigated the central and peripheral footprint of the major neurotrophin in the central nervous system, brain-derived neurotrophic factor (BDNF), on pain and mood perception of RA patients.

Methods: Pain symptomatology was assessed in 216 female RA patients (mean age $52 y$, mean disease duration 10 years) by a visual analogue scale (VAS), 18 tender points count (TPC), and by pressure-induced pain threshold measurement. The mood was patient-reported based on the Hospital Anxiety and Depression Scale (HADS). Clinical RA activity was assessed by DAS28. Serum levels of BDNF, IL6, IL1b, IL10 and IFN-gamma were measured by ELISA. Transcription of FOXO1 and FOXO3 was measured by RT-PCR in whole-blood RNA. Effect of BDNF signaling in leukocytes was assessed by differentially expressed gene (DEG) analysis in RNAseq of 24 female RA patients (R-studio, Bioconductor). High-resolution brain MRI was performed in a representative selection of 16 patients. Brain volumes were analyzed with MAPER software for accurate measurement of 83 anatomical regions (3) and compared between two groups of patients with high and low serum BDNF, respectively.

Results: In RA patients, high serum levels of BDNF were associated with low TPC (4.1 vs 5.3, $p=0.04$ ) and higher pain threshold ( $k P a, 416$ vs $382, p=0.09)$. No connection between BDNF and mood measures was evident. High BDNF was associated with high serum VEGF ( $p<0,001)$, IFNg $(p=0.0004), I L 1 b(p=0.036)$ and serum insulin $(p<0,001)$, but low resistin $(p=0.059)$. No correlation was found between BDNF with either serum IGF1 or inflammation parameters DAS28 and IL6. Serum BDNF was functional, since the RA patients with high BDNF had significantly larger brain volumes in specific regions and significantly lower FOXO1 mRNA in blood leukocytes ( $p=0.03)$. Specifically, structures of the limbic system, parahippocampus, lingual gyrus, nucleus accumbens and thalamus, key regions for the transmission of nociceptive information and central modulation of pain, were enlarged. BDNF production was measured in CD4-CD8- PBMC and was inversely related to expression of its high-affinity receptor TrkB in CD4+ PBMC. DEG analysis in CD4 T cells showed that low TrkB was associated with CD28+ transitional memory phenotype.

Conclusion: We conclude that high serum BDNF was associated with larger volumes of nociception-related brain regions and lower pain perception, acting independently of IGF1 and systemic inflammation.

References:

[1] Dougados M, Curr OppRheumatol 2016

[2] Andersson, Wasen, PNAS2017

[3] Heckemann, Neurolmage 2010

Disclosure of Interests: None declared

DOI: 10.1136/annrheumdis-2020-eular.5994

SAT0082

FRAX AND DAS IN MOROCCAN RHEUMATOID ARTHRITIS PATIENTS

H. Azzouzi ${ }^{1}$, O. Lamkhanat ${ }^{1}$, I. Linda ${ }^{1} .{ }^{1}$ Mohammed VI University Hospital, Rheumatology, Oujda, Morocco
Background: Rheumatoid Arthritis (RA) is one of the risk factors for the calculation of the 10 years fracture probability assessed by the FRAX tool.

Objectives: The aim was to study the association of disease activity and the 10 year fracture risk probability by the FRAX tool in our RA patients and their impact on fracture prevalence.

Methods: Cross-sectional study of the association FRAX and disease activity score (DAS 28 CRP) was designed. Patients with RA were included. Mean DAS was calculated for each patient adjusted on his follow-up duration. Data about patients (demographic, disease characteristics and fracture assessment) were collected. The 10 year fracture risk probability for major osteoporotic fracture was calculated with and without BMD (bone mineral density) using the FRAX tool for Morocco. Descriptive analysis and regressions were performed with SPSS.20. $\mathrm{p}<0.05$ was considered significant.

Results: One hundred and ninety nine RA patients were included with mean age of $55.5 \pm 12$ years. Women represented $91 \%$ and $40.1 \%$ had osteoporosis. Remission was observed in $86.4 \%$ with $95.5 \%$ taking methotrexate. $17.1 \%$ had vertebra fractures. FRAX and DAS were associated $(p=0.03)$, and both explained vertebral fracture (VF) prevalence. When adjusted on disease parameters, FRAX with and without BMD explained the vertebral prevalence $(p=0.02, O R=1.09[1.01$ 1.19]). However, age remains the only predictor of VF when adjusted on osteoporosis factors (DAS28CRP, menopause, BMI, smoking, diabetes, gender, steroid use, HAQ) and FRAX BMD.

Conclusion: Persistent disease activity was associated to high 10 year fracture risk probability calculated by the FRAX tool in RA.

Disclosure of Interests: None declared

DOI: 10.1136/annrheumdis-2020-eular.4467

\section{SAT0083 PREVALENCE OF DYSPHAGIA AND ASSOCIATED RISK FACTORS IN ELDERLY PATIENTS WITH RHEUMATOID ARTHRITIS}

T. Matsumoto ${ }^{1}$, S. Tsuboi ${ }^{1}$, T. Amano ${ }^{2}$. ${ }^{1}$ Shizuoka Welfare Hospital, Department of Rheumatology, Shizuoka, Japan; ${ }^{2}$ Shizuoka Welfare Hospital, Department of Orthopedic Surgery, Shizuoka, Japan

Background: Dysphagia (swallowing disorder) is an important health concern among the elderly that is associated with a poor prognosis [1]. Rheumatic dis eases such as dermatomyositis are thought to represent an important risk factor for dysphagia, but few studies have described the association between dysphagia and rheumatoid arthritis (RA), and details on the prevalence of dysphagia in RA patients is not known [2] [3]

Objectives: The present study aimed to determine the prevalence of dysphagia and associated risk factors among elderly patients with rheumatoid arthritis.

Methods: We conducted a cross-sectional study including 93 patients with RA and osteoarthritis (OA) over 65 years of age. OA patients were included in the study as healthy controls. Patients with a history of stroke, neuromuscular disease, or head and neck tumors were excluded from the study. From July to November 2019, the water swallowing test (WST) and repetitive saliva swallowing test (RSST) were performed to evaluate the presence or absence of dysphagia in the patients. We also checked oral conditions, hoarseness, temporomandibular joint symptoms, cervical range of motion limitations, and grip strength. In addition, interviews were conducted to investigate swallowing ability and aspiration history. We compared the prevalence of dysphagia between RA and OA patients and explored potential risk factors for dysphagia in RA patients using logistic regression models.

Results: Our study subjects comprised 63 RA patients (mean age, 73.8 years; $86.5 \%$ female) and 30 OA patients (mean age, 75.8 years; $82.3 \%$ female). The WST and RSST revealed that RA patients had a significantly higher prevalence of dysphagia than OA patients $(23.8 \%$ vs $6.7 \%, p<0.05)$. While RA patients with dysphagia $(n=15)$ were significantly older and had a longer disease duration than the OA patients, we observed no difference in disease activity or administrated drugs. Of the RA patients with dysphagia, $60 \%$ reported no previous episodes of aspiration. Increasing age (odds ratio (OR) 3.21 , 95\% confidence interval $(\mathrm{Cl})$ 1.06-4.56), cervical range of motion limitations (OR 3.14, 95\% Cl 1.02-7.24), opening disorder of the jaw (OR 2.26, 95\% Cl 1.12-4.86), and decreased grip strength (OR 1.96, 95\% Cl 1.01-4.15) were identified as factors related to the presence of dysphagia. Coexistence of Sjogren's syndrome did not significantly affect the prevalence of dysphagia.

Conclusion: Dysphagia was more prevalent among RA patients than in OA patients, suggesting an association with temporomandibular involvement, cervical disorder, and muscle weakness. Subclinical dysphagia should be assessed and monitored carefully in the clinical course of elderly patients with RA.

References:

[1] KW Altman et al. Consequence of dysphagia in the hospitalized patient: impact on prognosis and hospital resources. Arch Otolaryngol Head Neck Surg. 2010 Aug; 136 (8):784-9.

[2] Gilheaney Ó et al. The Prevalence of Oropharyngeal Dysphagia in Adults Presenting with Temporomandibular Disorders Associated with Rheumatoid 
Arthritis: A Systematic Review and Meta-analysis. Dysphagia. 2017 Oct; 32 (5):587-600.

[3] Mugii N et al. Oropharyngeal Dysphagia in Dermatomyositis: Associations with Clinical and Laboratory Features Including Autoantibodies. PLoS One. 2016 May 11;11 (5):e0154746.

Disclosure of Interests: None declared

DOI: 10.1136/annrheumdis-2020-eular.573

\section{\begin{tabular}{|l|l}
\hline SAT0084 SERUM ADIPOKINES PROFILE IN PATIENTS \\
SA
\end{tabular} WITH RHEUMATOID ARTHRITIS TREATED WITH TNF-INHIBITORS}

M. Novella-Navarro ${ }^{1,2}$, B. Hernández-Breijo ${ }^{2}$, F. Genre ${ }^{3}$, L. Lera-Gómez ${ }^{3}$, V. Pulito-Cueto ${ }^{3}$, L. Nuño ${ }^{1}$, A. Villalba ${ }^{1}$, A. Balsa ${ }^{1,2}$, C. Plasencia ${ }^{1,2} .{ }^{1} \mathrm{Hospital}$ Universitario La Paz, Madrid, Spain; ${ }^{2}$ Inmuno-Rheumatology Research Group IdiPaz, Madrid, Spain; ${ }^{3}$ Research Group on Genetic Epidemiology and Atherosclerosis in Systemic Diseases and in Metabolic Bone Diseases of the Musculoskeletal System. IDIVAL, Santander, Spain

Background: In recent years, the relationship between obesity and autoimmune diseases has taken interest, since adipose tissue has been identified as an endocrine organ that secretes cytokines (adipokines), among which leptin stands out as a soluble pro-inflammatory mediator associated with the body mass index (BMI).

Objectives: The main objectives of this study are: i) to analyse the influence of $\mathrm{BMI}$ on clinical response in Rheumatoid Arthritis (RA) patients who initiate TNF-inhibitor (TNFi) therapy; ii) to analyse the differences in the serum profile of adipokines (leptin and adiponectin) according to BMI and their association with response to treatment.

Methods: Observational study of a prospective cohort of 73 RA patients who initiated biological treatment with TNFi from the Complex Therapy Unit (CTU) of our Hospital. Patients were classified according to their BMI in normal-weight $(B M \mid<25)$ and overweight/obesity $(\mathrm{O} / \mathrm{O})(\mathrm{IMC} \geq 25)$. Demographic, clinical and laboratory variables were collected at baseline and at 6 months. Our outcome measures were DAS28-VSG remission (DAS28<2.6) at 6 months after TNFi initiation. Serum leptin and adiponectin levels were measured by Enzyme-Linked Immuno Sorbent Assay (ELISA) at baseline and 6 months. A descriptive sample analysis comparing the characteristics of both patient subgroups was performed using Chi-square, T-test for independent samples and U-Mann Whitney. Likewise, a bivariate analysis was carried out by means of binary logistic regression to assess the probable association of the parameters studied with remission.

Results: Of the 73 patients studied, $51 \%$ were classified in O/O group. The O/O patients presented higher levels of baseline CRP $(16.69 \pm 6.16$ vs $8.74 \pm 3.81$, $\mathrm{p}=0.01$ ). No statistically significant differences were observed in the remaining variables (sex, age at the beginning of the TNFi, disease duration, baseline DAS28 ), as well as therapeutic variables (use of previous DMARDs and doses of methotrexate and/or steroids). Patients with overweight/obesity presented higher DAS28-ESR values at 6 months of treatment $(3.59 \pm 1.14$ vs $2.93 \pm 1.27, p=0.02)$ and achieved remission less frequently $(18.9 \%$ vs $48.6 \%, p=0.007)$. Serum leptin levels were significantly higher in $\mathrm{O} / \mathrm{O}$ patients, both baseline $(29.39 \pm 21.50$ vs $13.49 \pm 8.78, p<0.001)$ and 6 months $(33.06 \pm 22.03$ vs $14.77 \pm 9.50, p<0.001)$ after TNFi initiation. In addition, O/O patients were less likely to reach remission at 6 months than normal-weight patients. [OR= 4.04 IC95\% $(1.40-11.64) ; p=0.009]$. Lower frequency of remission was associated to greater leptin levels at 6 months [OR=0.94 $\mathrm{Cl} 95 \%(0.90-098) ; p=0.012]$. No differences in serum adiponectin were found between both subgroups of patients.

Conclusion: In this RA patient cohort, overweight/obesity is associated with i) a reduced response to TNFi therapy and ii) a lower short-term remission rate. Within the adipokine profile, leptin seems to play a relevant role in the maintenance of pro-inflammatory activity with a negative influence on the response to TNFi therapy in O/O patients.

References:

[1] Versini M. et al. Autoimmun Rev. 2014; 13, 981-1000

[2] Toussirot E et al. Life Sci. 2015;140: 29-36.

Disclosure of Interests: Marta Novella-Navarro: None declared, Borja Hernández-Breijo: None declared, Fernanda Genre: None declared, Leticia LeraGómez: None declared, Verónica Pulito-Cueto: None declared, Laura Nuño: None declared, Alejandro Villalba: None declared, Alejandro Balsa Grant/ research support from: BMS, Roche, Consultant of: AbbVie, Gilead, Lilly, Pfizer, UCB, Sanofi, Sandoz, Speakers bureau: AbbVie, Lilly, Sanofi, Novartis, Pfizer, UCB, Roche, Nordic, Sandoz, Chamaida Plasencia: None declared DOI: 10.1136/annrheumdis-2020-eular.4458

\section{SAT0085 \\ METABOLIC SYNDROME AND ITS ASSOCIATION WITH RHEUMATIC DISEASES IN PARAGUAYAN PATIENTS}

A. Paats ${ }^{1}$, V. Valinotti ${ }^{1}$, R. Acosta ${ }^{1}$, L. Roman ${ }^{1}$, M. Duarte ${ }^{1}$, O. Centurion ${ }^{2}$, P. De Abreu ${ }^{3}$, T. Martinez ${ }^{4}$, G. Avila ${ }^{1}$, I. Acosta-Colman ${ }^{1}$, S. Cabrera-Villalba ${ }^{1}$.
${ }^{1}$ Clinicas Hospital, Universidad Nacional de Asuncion, Rheumatology, Asunción, Paraguay; ${ }^{2}$ Clinicas Hospital, Universidad Nacional de Asuncion, Cardiovascular Medicine, Asunción, Paraguay; ${ }^{3}$ Sociedad Paraguaya de Reumatología, Asunción, Paraguay; ${ }^{4}$ Laboratorio Curie, Asunción, Paraguay

Background: Metabolic syndrome (MS) is associated with increased abdominal adipose tissue and production of inflammatory cytokines. Patients with MS are at increased risk for developing cardiovascular disease and diabetes mellitus which are among the leading causes of death in chronic rheumatic diseases.

Objectives: To characterize patients with rheumatic disease and MS and its association with inflammatory markers.

Methods: Descriptive, cross sectional, prospective study, in 3 Paraguayan cohorts of patients with rheumatoid arthritis (RA), systemic sclerosis (SSc) and systemic lupus erythematosus (SLE). This study had two phases: the first one, included a standardized questionnaire according to the variables included in the Cardiovascular Risk project (PINV15-0346), from the Consejo Nacional de Ciencias y Tecnología (CONACYT), and physical examination; the second one included laboratory sample collection performed by a specialized laboratory for serum biomarkers measurement for cardiovascular risk prediction (i.e endothelin alpha-TNF, E-selectin, t-PA, VCAM, PAI-1 and high sensitivity-CRP levels). MS patients were categorized according to 2007 ALAD criteria. All patients signed informed consent. SPSS Statistics v23 was used for data analysis. Quantitative variables were presented as means and qualitative variables as frequencies. Chi square test was performed for comparisons between dichotomous variables. A p value $\leq 0.05$ was used for statistical significance.

Results: We included a total of 253 patients, 100 with RA, 100 with SLE and 52 with SSc. Metabolic syndrome was found in 23,58\% (50/212). There was no significant difference in MS prevalence between diseases, but there was a higher frequency of increased abdominal circumference in RA and low HDL in SLE. Frequencies for different features of MS in RA, SLE and SSc are detailed in table 1.

Table 1. Frequencies of MS component in SLE, SSc and RA.

\begin{tabular}{lcccc}
\hline & SLE & SSc & RA & $p$ \\
\hline Abdominal circumference criteria & $43,4 \%(43 / 99)$ & $46,66 \%(21 / 46)$ & $64 \%(64 / 100)$ & 0,009 \\
\hline Hypertension criteria & $67 \%(67 / 100)$ & $67,3 \%(35 / 52)$ & $55 \%(55 / 100)$ & 0,152 \\
HDL criteria & $55,22 \%(37 / 67)$ & $52,63 \%(20 / 38)$ & $16,12 \%(15 / 93)$ & 0,000 \\
TAG criteria & $22,38 \%(15 / 67)$ & $28,94 \%(11 / 38)$ & $22,58 \%(21 / 93)$ & 0,703 \\
Glycemia criteria & $7,69 \%(4 / 52)$ & $13,15 \%(5 / 38)$ & $25,67 \%(19 / 74)$ & 0,114 \\
Metabolic Syndrome & $24,35 \%(19 / 78)$ & $25 \%(9 / 36)$ & $22,44 \%(22 / 98)$ & 0,934
\end{tabular}

Regarding inflammatory biomarkers, there was a significant difference between biomarkers elevated in each disease: hsCRP was found more frequently in RA, E-Selectin in SSc and elevated in each disease: hsCRP was found
VCAM and PAI-1 were more prevalent in SLE.

Table 2. Frequency of high serum inflammatory biomarkers in SLE, RA and SSc.

\begin{tabular}{lcccc}
\hline & SLE & SSc & RA & p \\
\hline hsCRP & $23,52 \%(16 / 68)$ & $26,31 \%(10 / 38)$ & $42,85 \%(36 / 84)$ & 0,027 \\
E-Selectin & $5,88 \%(4 / 68)$ & $21,05 \%(8 / 38)$ & $5,95 \%(5 / 84)$ & 0,014 \\
t-PA & $0 \%(0 / 68)$ & $2,63 \%(1 / 38)$ & $5,95 \%(5 / 84)$ & 0,111 \\
VCAM & $20,58 \%(14 / 68)$ & $8,1 \%(3 / 37)$ & $0 \%(0 / 83)$ & 0,000 \\
TNF-a & $7,35 \%(5 / 68)$ & $18,42 \%(7 / 38)$ & $13,09 \%(11 / 84)$ & 0,229 \\
Endotelin & $20,58 \%(14 / 68)$ & $31,57 \%(12 / 38)$ & $19,75 \%(16 / 81)$ & 0,192 \\
PAl-1 & $11,36 \%(5 / 44)$ & $0 \%(0 / 38)$ & $0 \%(0 / 83)$ & 0,002 \\
\hline
\end{tabular}

Conclusion: We found a similar frequency of metabolic syndrome in our cohorts of RA, SSc and SLE Paraguayan patients but they had a different clinical and serological profile, suggesting that the pathways leading to metabolic syndrome are dissimilar in each disease. We need more studies to confirm this hypothesis Disclosure of Interests: None declared

DOI: 10.1136/annrheumdis-2020-eular.6121

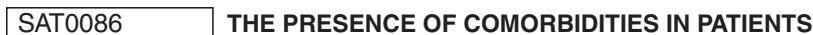 WITH RHEUMATOID ARTHRITIS IS ASSOCIATED WITH BAD PATIENT-REPORTED OUTCOMES (PROS)}

I. Pereira ${ }^{1}$, T. Coan ${ }^{1}$, G. Castro ${ }^{1}$, G. Castelar ${ }^{2}$, A. B. Vargas-Santos ${ }^{2}$,

C. Albuquerque ${ }^{3}$, A. P. Gomides ${ }^{3}$, M. Bertolo ${ }^{4}$, P. Louzada Jr ${ }^{5}$, R. Giorgi ${ }^{6}$,

S. Radominsky ${ }^{7}$, M. F. Guimarães ${ }^{8}$, K. Bonfiglioli ${ }^{9}$, M. D. F. Sauma ${ }^{10}$,

C. Brenol ${ }^{11}$, E. Coutinho ${ }^{12}$, L. Mota ${ }^{3} .{ }^{1}$ Universidade do Sul de Santa Catarina, Palhoça, Brazil; ${ }^{2}$ UERJ, Rio de Janeiro, Brazil; ${ }^{3}$ UnB, Brasilia, Brazil; ${ }^{4}$ Unicamp, Campinas, Brazil; ${ }^{5}$ USP, Ribeirão Preto, Brazil; ${ }^{6} \mathrm{Hospital}$ do Servidor Público Estadual de São Paulo, São Paulo, Brazil; ${ }^{7}$ UFPr, Curitiba, Brazil; ${ }^{8}$ UFMG, Belo Horizonte, Brazil; ${ }^{9}$ USP, São Paulo, Brazil; ${ }^{10}$ UFPA, Belém, Brazil; ${ }^{11}$ UFRGS, Porto Alegre, Brazil; ${ }^{12}$ Fundação Oswaldo Cruz, Rio de Janeiro, Brazil 